\title{
Erratum to: Shape distribution-based retrieval of 3D CAD models at different levels of detail
}

\author{
Hyungki Kim ${ }^{1} \cdot$ Moohyun Cha $^{1} \cdot$ Duhwan Mun $^{2}$
}

Published online: 7 September 2017

(C) Springer Science+Business Media, LLC 2017

Erratum to: Multimed Tools Appl (2016) 76:15867-15884

https://doi.org/10.1007/s11042-016-3881-5

In the original publication, the corresponding author's email address (Duhwan Mun) and Fig. 1 image were incorrect. The correct email address is given below and the correct image of Fig. 1 is also presented below.

The online version of the original article can be found at https://oi.org/10.1007/s11042-016-3881-5

Duhwan Mun

dhmun@knu.ac.kr

Hyungki Kim

diskhkme@gmail.com

Moohyun Cha

mhcha@kimm.re.kr

1 Mechanical Systems Safety Research Division, Korea Institute of Machinery and Materials, 156, Gajeongbuk-ro, Yuseong-gu, Daejeon 305-701, South Korea

2 Department of Precision Mechanical Engineering, Kyungpook National University,

Gyeongsangbuk-do, South Korea 


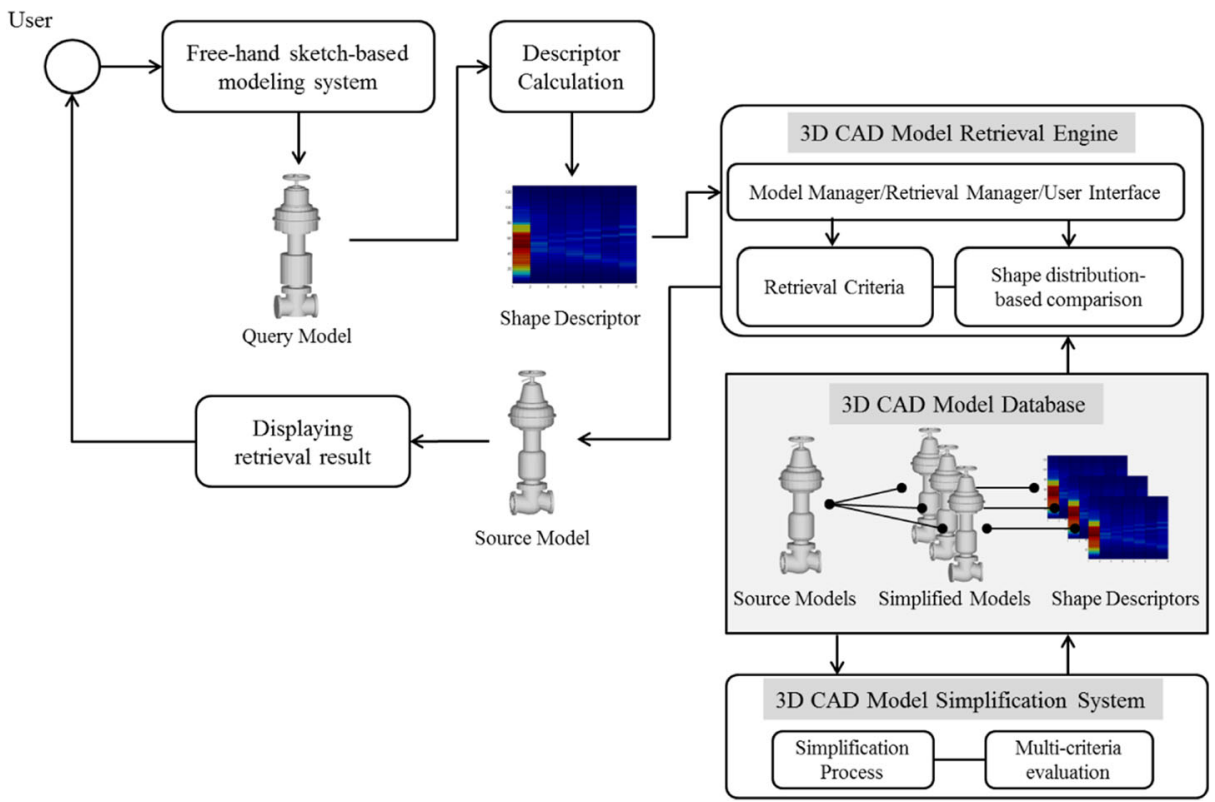

